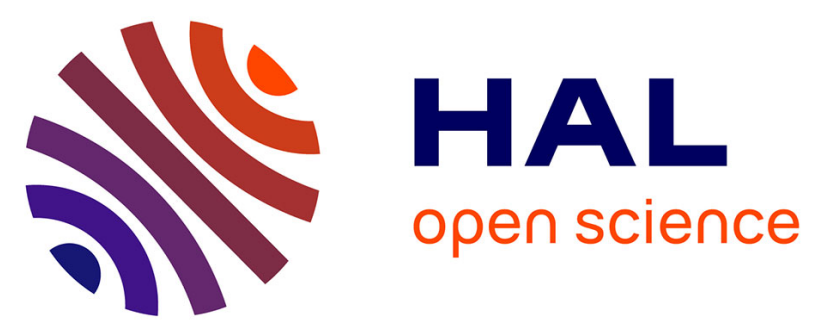

\title{
Synthèse, caractérisations et tests photocatalytiques d'un matériau argileux d'origine naturelle à base de beidellite fonctionnalisée par TiO2
}

Lahcen Bouna, Benaïssa Rhouta, M'Barek Amjoud, Francis Maury, Amane

Jada, Lahcen Daoudi, François Senocq, Marie-Christine Lafont, Christophe

Drouet

\section{To cite this version:}

Lahcen Bouna, Benaïssa Rhouta, M'Barek Amjoud, Francis Maury, Amane Jada, et al.. Synthèse, caractérisations et tests photocatalytiques d'un matériau argileux d'origine naturelle à base de beidellite fonctionnalisée par TiO2. Matériaux \& Techniques, 2012, vol. 100, pp. 241-252. 10.1051/mattech/2012033 . hal-00864847

\section{HAL Id: hal-00864847 https://hal.science/hal-00864847}

Submitted on 23 Sep 2013

HAL is a multi-disciplinary open access archive for the deposit and dissemination of scientific research documents, whether they are published or not. The documents may come from teaching and research institutions in France or abroad, or from public or private research centers.
L'archive ouverte pluridisciplinaire HAL, est destinée au dépôt et à la diffusion de documents scientifiques de niveau recherche, publiés ou non, émanant des établissements d'enseignement et de recherche français ou étrangers, des laboratoires publics ou privés. 


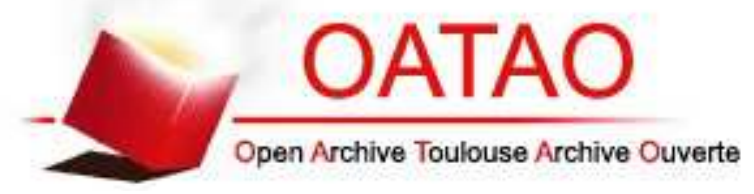

\section{Open Archive TOULOUSE Archive Ouverte (OATAO)}

OATAO is an open access repository that collects the work of Toulouse researchers and makes it freely available over the web where possible.

This is an author-deposited version published in : http://oatao.univ-toulouse.fr/ Eprints ID : 8753

To link to this article : doi:10.1051/mattech/2012033

URL : http://dx.doi.org/10.1051/mattech/2012033

To cite this version : Bouna, Lahcen and Rhouta, Benaïssa and Amjoud, M'barek and Maury, Francis and Jada, Amane and Daoudi, Lahcen and Senocq, François and Lafont, Marie-Christine and Drouet, Christophe Synthèse, caractérisations et tests photocatalytiques d'un matériau argileux d'origine naturelle à base de beidellite fonctionnalisée par TiO2. (2012) Matériaux \& Techniques, vol. $100\left(\mathrm{n}^{\circ}\right.$ 3). pp. 241-252. ISSN 0032-6895

Any correspondance concerning this service should be sent to the repository administrator: staff-oatao@ listes-diff.inp-toulouse.fr 


\title{
Synthèse, caractérisations et tests photocatalytiques d'un matériau argileux d'origine naturelle à base de beidellite fonctionnalisée par $\mathrm{TiO}_{2}$
}

\author{
L. Bouna ${ }^{1,2}$, B. Rhouta1, M. Amjoud ${ }^{1}$, F. Maury², A. Jada ${ }^{3}$, L. Daoudi ${ }^{4}$, \\ F. Senocq ${ }^{2}$, M.-C. Lafont ${ }^{2}$ et C. Drouet $^{2}$ \\ 1 Laboratoire de Matière Condensée et Nanostructures (LMCN), Université Cadi Ayyad, BP 549, \\ Marrakech, Maroc \\ e-mail : rhoutab@yahoo.fr \\ 2 CIRIMAT, ENSIACET, 4 Allée Emile Monso, BP 44362, 31432 Toulouse Cedex 4, France \\ 3 Institut de Science des Matériaux de Mulhouse (IS2M), 15 rue Jean Starcky, BP 2488, \\ 68057 Mulhouse Cedex, France \\ 4 Laboratoire de Géosciences et Géoenvironnement (LGG), Université Cadi Ayyad, BP 549, Marrakech, \\ Maroc
}

Mots-clés :

Beidellite; $\mathrm{TiO}_{2}$; photocatalyse ; orange $\mathrm{G}$; nanomatériaux

Key words:

Beidellite; $\mathrm{TiO}_{2}$; photocatalysis; orange G; nanomaterials

\begin{abstract}
Résumé - Cette étude porte sur la fonctionnalisation par $\mathrm{TiO}_{2}$ d'une argile marocaine de type beidellite et sur l'évaluation de son activité photocatalytique pour l'élimination d'un colorant anionique : I'orange G (OG), utilisé dans l'industrie textile. Les échantillons argileux ont été caractérisés, avant et après fonctionnalisation, par DRX, MEB et MET couplé à l'analyse EDX, TG-ATD, FTIR, ICP et RMN du solide. La beidellite est aluminifère, dioctaédrique, de formule chimique $\left(\mathrm{Si}_{7,57} \mathrm{Al}_{0,43}\right)_{8}\left(\mathrm{Al}_{2,75} \mathrm{Fe}_{0,73} \mathrm{Mg}_{0,38} \mathrm{Ti}_{0,14}\right)_{4}\left(\mathrm{Na}_{0,37} \mathrm{~K}_{0,23} \mathrm{Mg}_{0,01}\right)$ avec une CEC, une surface spécifique et un volume poreux total de l'ordre de $48 \mathrm{méq} / 100 \mathrm{~g}, 72 \mathrm{~m} / \mathrm{g}$ et $0,128 \mathrm{~cm}^{3} / \mathrm{g}$ respectivement. Les matériaux argileux nanocomposites obtenus sont constitués de nanoparticules de $\mathrm{TiO}_{2}$ (taille moyenne $10 \mathrm{~nm}$ ) supportées par les feuillets de la beidellite. Leurs surfaces spécifiques sont quasiment du même ordre de grandeur que celle de la beidellite tandis que leurs volumes poreux augmentent pour atteindre $0,226 \mathrm{~cm}^{3} / \mathrm{g}$. L'échantillon calciné à $600{ }^{\circ} \mathrm{C}$, dans lequel $\mathrm{TiO}_{2}$ est sous forme d'anatase, manifeste une activité photocatalytique remarquable vis-à-vis de l'élimination de l'OG en milieu aqueux.
\end{abstract}

\begin{abstract}
Synthesis, characterization and photocatalytic tests of beidellite based natural clay mineral functionalized by $\mathrm{TiO}_{2}$. This study deals with the preparation of $\mathrm{TiO}_{2}$ functionalized Moroccan beidellite clay mineral and the evaluation of its photocatalytic activity toward the removal of anionic dye Orange G (OG) largely used in textile industry. The clayey materials were characterized before and after functionalization using XRD, SEM, TEM equipped with EDX, TG-DTA, FTIR, ICP and MAS-NMR techniques. The beidellite was a dioctahedral alumino-ferruginous whose structural formula was $\left(\mathrm{Si}_{7.57} \mathrm{Al}_{0.43}\right)_{8}$ $\left(\mathrm{Al}_{2.75} \mathrm{Fe}_{0.73} \mathrm{Mg}_{0.38} \mathrm{Ti}_{0.14}\right)_{4}\left(\mathrm{Na}_{0.37} \mathrm{~K}_{0.23} \mathrm{Mg}_{0.01}\right)$ and exhibited a CEC, a specific surface area and a total porous volume around $48 \mathrm{meq} / 100 \mathrm{~g}, 72 \mathrm{~m}^{2} / \mathrm{g}$ and $0.128 \mathrm{~cm}^{3} / \mathrm{g}$, respectively. The clayey nanocomposites materials obtained were made up of $\mathrm{TiO}_{2}(10 \mathrm{~nm})$ supported onto beidellite layers. Their specific surface areas were quite similar to those of the starting beidellite whilst their porous volumes increased to reach $0.226 \mathrm{~cm}^{3} / \mathrm{g}$. The sample annealed at $600^{\circ} \mathrm{C}$, in which $\mathrm{TiO}_{2}$ crystallized in the form of anatase, was the best photocatalyst toward the removal of OG from aqueous media.
\end{abstract}

oxydation photocatalytique hétérogène émerge comme une technique alternative efficiente dans le domaine des traitements des eaux usées industrielles [1-5]. Le principe de cette technique repose sur la création des espèces réactives trous $\left(\mathrm{h}^{+}\right)$et/ou radical hydroxyl $\left({ }^{\bullet} \mathrm{OH}\right)$ en irradiant un oxyde semi-conducteur avec une source d'énergie $(h v)$ supérieure à son gap d'énergie $[1,5]$. Les espèces réactives ainsi formées sont capables d'induire la minéralisation totale des polluants organiques 
en $\mathrm{CO}_{2}$ et $\mathrm{H}_{2} \mathrm{O}$ [1]. $\mathrm{TiO}_{2}$ sous forme anatase est l'oxyde semi-conducteur le plus actif en photocatalyse et le plus utilisé en raison de sa non nocivité, de son faible coût et de son inertie chimique [1]. Cependant, son utilisation sous forme de poudre nanométrique (e.g., Degussa P25) pose des problèmes d'une part en raison de l'agglomération des particules en cours de service, ce qui réduit son activité photocatalytique, et d'autre part car son élimination du milieu aqueux nécessite une microfiltration coûteuse $[1,4,6,7]$.

Des travaux de recherches ont récemment visé à immobiliser $\mathrm{TiO}_{2}$ sur un support [6-14]. Parmi les supports envisagés, les minéraux argileux, en l'occurrence ceux de la famille des smectites, sont considérés prometteurs. En effet, en raison de leurs caractéristiques (structures lamellaires, faible charge des feuillets) et de leurs propriétés d'adsorption [15], ces minéraux se prêtent à la modification chimique par pontage en procédant par intercalation par échange cationique des cations interfoliaires compensateurs de charge avec des polycations métalliques suivie d'un traitement thermique permettant leur conversion en piliers d'oxydes métalliques (argiles à piliers PILCs) $[6,16]$. La préparation d'argiles à piliers de $\mathrm{TiO}_{2}$ s'est avérée une solution inapplicable d'une part en raison des conditions $\mathrm{d}$ 'hydrolyse très acides nécessitées pour la formation du sol des polycations de titane et qui sont très sévères vis-à-vis des minéraux argileux smectites, et d'autre part de la faible cristallinité associée à la faible activité photocatalytique des piliers de $\mathrm{TiO}_{2}$ dans le cas où ils seraient formés [7]. Pour pallier à ces inconvénients, des travaux très récents $[12,14]$ suggèrent une méthode colloïdale mettant en jeu la modification préalable du minéral argileux par un surfactant cationique, jouant le rôle d'agent directeur, qui assure un environnement organophile favorable à l'ancrage par voie sol-gel des particules de $\mathrm{TiO}_{2}$ aux particules d'argiles. Aranda et al. [14] ont de plus montré que le succès de cette méthode de fonctionnalisation est tributaire de la nature du minéral argileux.

Cette étude vise à explorer la possibilité d'appliquer cette méthode colloïdale à un minéral argileux de type smectite, nommé- ment la beidellite, isolée d'une argile naturelle marocaine prélevée dans le Haut Atlas près d'Agadir pour préparer des photocatalyseurs supportés efficaces.

\section{Techniques expérimentales}

\subsection{Origine et purification de l'argile}

Des échantillons de l'argile, désignée TAG, ont été prélevés dans la partie occidentale du Haut Atlas, dans la localité de Taghazout (bassin d'Agadir). Ils ont été d'abord broyés, tamisés à $50 \mu \mathrm{m}$ et traités avec une solution tampon $\left(\mathrm{CH}_{3} \mathrm{COOH}, \mathrm{CH}_{3} \mathrm{COONa}\right)$ de $\mathrm{pH} 4,5$. La fraction argileuse quasi pure homoionisée avec $\mathrm{Na}^{+}$(désignée $\mathrm{Na}^{+}$-TAG) a été récupérée selon la procédure que nous avons décrite ailleurs [17].

\subsection{Préparation du nanocomposite photocatalyseur supporté $\mathrm{TiO}_{2}$-beidellite}

La synthèse du matériau photocatalyseur supporté $\mathrm{TiO}_{2}$-beidellite a été réalisée selon la méthode colloïdale décrite en détail ailleurs [14]. Une suspension aqueuse de l'échantillon $\mathrm{Na}^{+}$-TAG $(3 \mathrm{~g}$ dans $300 \mathrm{ml}$ d'eau) a été d'abord échangée à $50{ }^{\circ} \mathrm{C}$ sous agitation durant $48 \mathrm{~h}$ avec $0,2 \mathrm{~g}$ de surfactant cationique (bromure de céthyltriméthylammonium, CTAB) pour former l'argile organophile désignée comme CTA ${ }^{+}$-TAG. Ensuite, $5 \mathrm{ml}$ d'isopropoxyde de titane $\left(\mathrm{Ti}(\mathrm{O}-\mathrm{i}-\mathrm{Pr})_{4}\right)$ ont été ajoutés à une suspension de $1 \mathrm{~g}$ de $\mathrm{CTA}^{+}-\mathrm{TAG}$ dans $7 \mathrm{ml}$ d'isopropanol. Finalement, quelques gouttes d'eau ont été lentement ajoutées sous agitation jusqu'à la formation spontanée d'un gel. L'échantillon du gel précurseur brut d'élaboration obtenu (désigné CTA+-TAG-Ti) est séché pendant 2 jours à $60^{\circ} \mathrm{C}$ puis calciné sous air pendant $1 \mathrm{~h}$ à différentes températures. Les échantillons ainsi obtenus sont référencés Ti-TAG-T, où $T$ désigne la température de maintien isotherme.

\subsection{Techniques de caractérisations}

La Capacité d'Échange Cationique (CEC) de la fraction argileuse ( $\left.\mathrm{Na}^{+}-\mathrm{TAG}\right)$ a été évaluée en procédant au dosage des particules 
d'argiles chargées négativement à l'aide des molécules de surfactant cationique (CTAB). Le point de charge nulle (PCN) a été déterminé à partir de la courbe représentant la variation du potentiel d'écoulement induit (PEI) en fonction de la quantité du surfactant CTAB ajoutée. Les mesures du PEI ont été menées moyennant l'instrument micro mütek de type Mütek PCD 02 dont le principe est exhaustivement décrit par Jada et al. [18]. Brièvement, La cellule de mesure est composée d'une enceinte cylindrique en poly(tétrafluoroéthylène) (PTFE) comportant un piston en PTFE. $10 \mathrm{ml}$ d'une suspension d'argile ( $0,2 \%$ pds) sont placés dans l'espace $(0,5 \mathrm{~mm})$ compris entre la paroi de l'enceinte et le piston et sont dispersés sous ultrasons pendant 2 min. Après avoir relevé le $\mathrm{pH}$ initial, des quantités variables de solution aqueuse du titrant sont ajoutées, au bout de chaque minute, à cette suspension à l'aide d'une micropipette BRAND. Le potentiel d'écoulement induit (PEI) est mesuré entre deux électrodes en or placées aux extrémités opposées de l'enceinte. Au fil des mesures, le piston se déplace sinusoïdalement à une fréquence de $4 \mathrm{~Hz}$ en va et vient et force la suspension d'argile à se déplacer et à s'écouler le long des parois de l'enceinte. Le potentiel normé, mesuré durant le mouvement du piston, résulte de la séparation des contre ions des particules d'argiles adsorbées sur les parois de l'enceinte.

Les compositions chimiques des différents échantillons préparés ont été déterminées par ICP en utilisant un appareil Serie X2 de Termo Electron équipé d'un nébuliseur Meinhard et d'un détecteur de type simulscan ETP. Pour déterminer la teneur en Ti, on a utilisé les isotopes ${ }^{47} \mathrm{Ti}$ et ${ }^{49} \mathrm{Ti}$ avec une technologie de cellule de collision. La surface spécifique a été mesurée par BET à partir des isothermes d'adsorption-désorption de $\mathrm{N}_{2}$ à $77 \mathrm{~K}$ obtenue à l'aide d'un appareil ASAP 2020 V 3.01 H de Micromeritics.

Les différents échantillons ont été caractérisés, avant et après fonctionnalisation par $\mathrm{TiO}_{2}$, par diffraction des rayons $X(\mathrm{DRX})$, analyse thermique (ATG-ATD), spectroscopie Infra rouge (IR), microscopies électroniques à balayage (MEB) et en transmission (MET). Les diffractogrammes de rayons $X$ ont été enregistrés dans l'intervalle angu- laire $2 \theta=2^{\circ}-60^{\circ}$ à l'aide d'un appareil Seifert XRD 3000TT (raie $\mathrm{Cu} \mathrm{K}_{\alpha}$; équipé d'un monochromateur arrière en graphite) dans la configuration Bragg-Brentano. Les thermogrammes TG et ATD ont été collectés à l'aide d'un appareil Labsys de Setaram sur des masses d'échantillon d'environ $60 \mathrm{mg}$ et selon une rampe de température de $10 \mathrm{~K} / \mathrm{min}$ de l'ambiante jusqu'à 1273 K. Les observations microstructurales ont été faites avec un MEB Jeol JSM 6400 et un MET Jeol JEM 2010 équipé d'un analyseur EDX (TRACOR).

\subsection{Essais d'activité photocatalytique}

Les activités photocatalytiques des différents échantillons préparés ont été testées vis-à-vis de l'élimination de l'orange G (OG) en utilisant un protocole précédemment décrit [19]. Les différentes caractéristiques de ce colorant, connu d'après la nomenclature sous le nom d'acide 7-Hydroxy8-(phenylazo)-1,3-naphthalenedisulfonic, sel de disodium, sont consignées dans le tableau 1.

La figure 1 montre la formule développée du colorant OG et son spectre d'absorption. L'absorbance maximale de ce colorant est à 480 nm. Le composé utilisé dans cette étude est fourni par Aldrich et présente une pureté de $90 \%$. Le choix de ce colorant anionique est justifié, par son adsorption négligeable sur les minéraux argileux en raison des répulsions électrostatiques entre les particules argileuses chargées négativement et les entités anioniques du colorant [20]. Une masse de $25 \mathrm{mg}$ de catalyseur est dispersée dans $25 \mathrm{ml}$ de solution aqueuse d'orange $\mathrm{G}$ de concentration $10^{-5} \mathrm{M}$ et de $\mathrm{pH} 7$, puis le mélange a été placé dans une cuve en quartz à l'abri de la lumière et sous agitation pendant 30 min afin de permettre à l'équilibre d'adsorption des espèces sur la surface de s'établir. La suspension est ensuite illuminée sous agitation par une lampe UV (Philips HPN) de $125 \mathrm{~W}$ dont la principale radiation est à 365 nm, ce qui correspond à une énergie d'excitation d'environ $3,4 \mathrm{eV}$, légèrement plus élevée que le gap d'énergie de l'anatase $(3,2 \mathrm{eV})$, et au minimum d'absorption du colorant ce qui évite sa photolyse. La position du réacteur a été ajustée par rapport 
Tableau 1. Caractéristiques du colorant Orange G utilisé en tant que polluant modèle dans les tests de photocatalyse.

Table 1. Characteristics of the Orange $G$ dye used as a model pollutant in the photocatalysis tests.

\begin{tabular}{cccccc}
\hline $\begin{array}{c}\text { Nom du } \\
\text { Colorant }\end{array}$ & $\begin{array}{c}\text { Concentration } \\
\text { initiale }(\mathrm{M})\end{array}$ & $\begin{array}{c}\text { Masse molaire } \\
(\mathrm{g} / \mathrm{mol})\end{array}$ & $\begin{array}{c}\text { Formule } \\
\text { moléculaire }\end{array}$ & $\mathrm{pKa}$ & $\begin{array}{c}\text { Domaine } \\
\text { d'application }\end{array}$ \\
\hline Orange $\mathrm{G}$ & $10^{-5}$ & 452,38 & $\mathrm{C}_{16} \mathrm{H}_{10} \mathrm{~N}_{2} \mathrm{Na}_{2} \mathrm{O}_{7} \mathrm{~S}_{2}$ & 11,5 & industrie textile \\
\hline
\end{tabular}

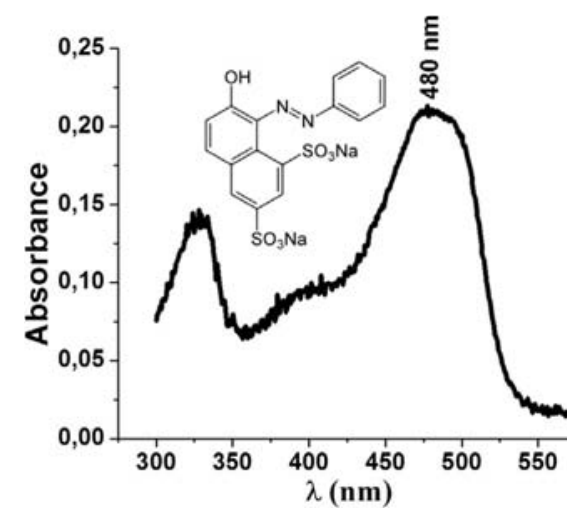

Fig. 1. Spectre d'absorption d'une solution aqueuse d'orange $\mathrm{G}\left(10^{-5} \mathrm{M}\right)$ et formule développée du colorant.

Fig. 1. Absorption spectra of Orange G aqueous solution $\left(10^{-5} \mathrm{M}\right)$ and developed formula of the dye.

à la lampe UV de sorte que le flux d'irradiation, contrôlé par un radiomètre, soit de $1,05 \mathrm{~mW} / \mathrm{cm}^{2}$ et donc de l'ordre de grandeur de l'intensité du spectre solaire sur la terre [21]. Le suivi de la réaction photocatalytique se fait par photocolorimétrie en prélevant, au bout de chaque $15 \mathrm{~min}, 2 \mathrm{~mL}$ de la suspension argile/solution de l'OG. Les particules argileuses ont été éliminées avec un filtre en membrane PTFE dont le seuil de coupure est de $0,45 \mu \mathrm{m}$ et le surnageant récupéré est analysé par le biais d'un spectrophotomètre UV / Vis / NIR. A cet effet, la concentration de l'OG dans le surnageant a été déterminée en reportant l'absorbance correspondante mesurée à $480 \mathrm{~nm}$ dans la courbe d'étalonnage préétablie et en appliquant la loi de Beer-Lambert.

\section{Résultats et discussions}

\subsection{Caractérisations des différents échantillons avant et après fonctionnalisations}

La figure 2 représente la superposition des diffractogrammes de l'argile brute (TAG) et

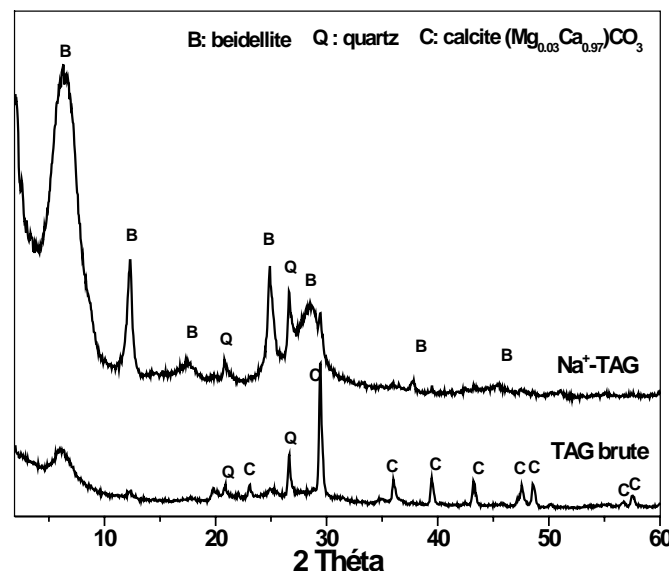

Fig. 2. Diffractogrammes $R X$ de l'argile brute (TAG) et de sa fraction fine isolée ( $\left.\mathrm{Na}^{+}-\mathrm{TAG}\right)$.

Fig. 2. X ray diagrams of raw clay (TAG) and its isolated fine fraction $\left(\mathrm{Na}^{+}-\mathrm{TAG}\right)$.

de sa fraction fine pure isolée $\left(\mathrm{Na}^{+}-\mathrm{TAG}\right)$. À l'état brut de l'argile, on remarque des pics à $2 \theta=6,27^{\circ}, 12,27^{\circ}$ et $24,82^{\circ}$ correspondant respectivement aux distances basales $d_{001}=1,41 \mathrm{~nm}, d_{002}=0,72 \mathrm{~nm}$ et $d_{004}=0,36 \mathrm{~nm}$ et qui sont caractéristiques du minéral argileux. De même, on remarque la présence d'impuretés de quartz (pics à $2 \theta=20,76^{\circ}$ et $\left.26,58^{\circ}\right)$ et de calcite $(2 \theta=$ $23,07^{\circ} ; 29,44^{\circ} ; 35,99^{\circ} ; 39,5^{\circ} ; 43,19^{\circ}, 47,53$ et $48,54^{\circ}$ ). À l'état purifié, on remarque l'intensification des raies caractéristiques du minéral argileux et la subsistance des pics du quartz. En revanche, On note une diminution importante des intensités des pics de diffraction caractéristiques de la calcite traduisant sa disparition après le traitement par une solution tampon définie ci-dessus dans le paragraphe 1.1.

Pour déterminer la nature du minéral argileux, des traitements spécifiques par saturation à l'éthylène glycol et de recuit ont été effectués sur la fraction argileuse $\left(\mathrm{Na}^{+}-\right.$ TAG) extraite. La figure 3 représente la superposition des diffractogrammes ainsi obtenus dans $l^{\prime}$ intervalle angulaire $\left[2^{\circ}-15^{\circ}\right]$. 


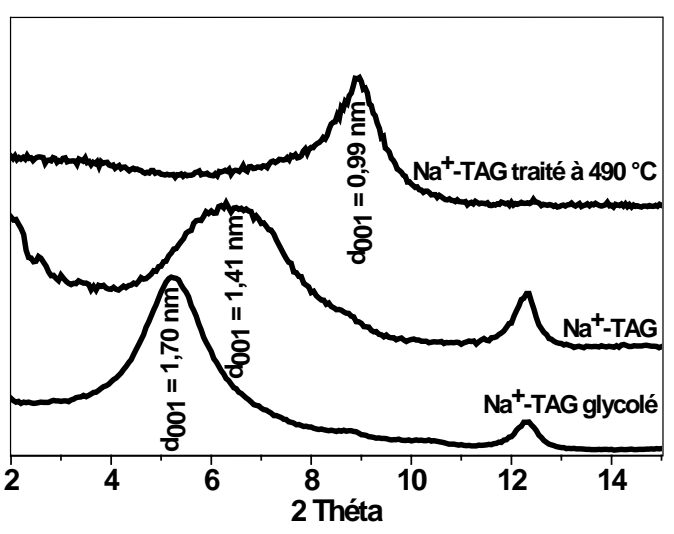

Fig. 3. Diffractogrammes aux petits angles de la fraction argileuse fine isolée après les différents traitements spécifiques.

Fig. 3. X ray diagrams recorded in small angles region on isolated fine clay fraction upon different specific treatments.

On constate que la raie principale du minéral argileux, correspondant à la distance basale $d_{001}=1,41 \mathrm{~nm}$, se déplace vers les petits angles $\left(d_{001}=1,70 \mathrm{~nm}\right)$ suite à son gonflement par incorporation des molécules d'éthylène glycol dans ses espaces interfoliaires. Par ailleurs, le traitement thermique déplace la raie vers les grands angles $\left(d_{001}=0,99 \mathrm{~nm}\right)$ en raison de la déshydratation des cations interfoliaires et de l'affaissement de la structure du minéral argileux. Ces résultats révèlent la sensibilité du minéral argileux aux traitements spécifiques et témoignent de son caractère smectite [22]. La nature diocataédrique ou trioctaédrique du minéral argileux peut être identifiée à partir de la valeur du paramètre $b$ en effectuant l'acquisition des diffractogrammes dans l'intervalle angulaire $\left[58^{\circ}-66^{\circ}\right]$ correspondant à la réflexion (060) [22]. Les minéraux argileux trioctaédriques présentent des paramètres $b$ supérieurs à $0,91 \mathrm{~nm}$ alors que les minéraux diocatédriques sont caractérisés par une valeur de $b$ inférieure. Le diffractogramme enregistré pour l'échantillon $\mathrm{Na}^{+}$-TAG sur la poudre désorientée de l'échantillon $\mathrm{Na}^{+}$TAG (Fig. 4) révèle la réflexion (060) correspondant à $d_{060}=0,15 \mathrm{~nm}$ et conduisant à un paramètre $b=0,90 \mathrm{~nm}$. Cette valeur inférieure à $0,91 \mathrm{~nm}$ confirme que le minéral argileux smectite composant l'argile TAG est de type dioctaédrique [23], et donc que la composition de sa couche octaédrique est

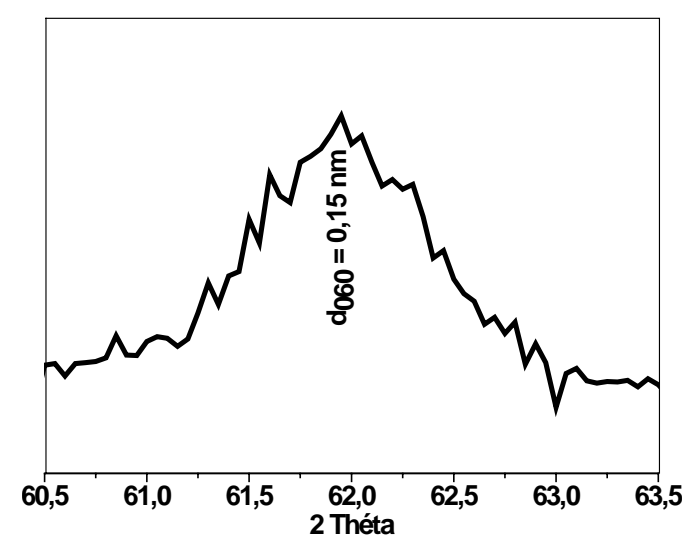

Fig. 4. Diffractogramme de la fraction argileuse fine isolée ( $\mathrm{Na}^{+}$-TAG) dans la région de diffraction (060).

Fig. 4. X ray diagram recorded on isolated fine clay fraction in the (060) diffraction region.

prédominée par la présence de cations trivalents $\mathrm{Al}^{3+}$ et $\mathrm{Fe}^{3+}$.

Les thermogrammes TG-ATD relatifs à l'argile brute TAG et sa fraction fine argileuse $\left(\mathrm{Na}^{+}-\mathrm{TAG}\right)$ sont reportés dans la figure 5. On distingue dans les deux cas un phénomène endothermique dont le maximum est à $115^{\circ} \mathrm{C}$ environ, associé à une perte de masse de l'ordre de 6,8\%. Ceci est attribué à l'élimination de l'eau physisorbée sur les surfaces des particules et/ou d'hydratation des cations interfoliaires. Un autre pic endothermique est observé à environ $510{ }^{\circ} \mathrm{C}$. Il s'accompagne d'une perte de poids de $4,26 \%$ et correspond à la déshydroxylation du minéral argileux. Parmi les variants composant la famille des minéraux argileux smectites, ce pic est caractéristique de la beidellite [24]. Un autre pic endothermique, rencontré aux alentours de $790{ }^{\circ} \mathrm{C}$ environ, parait plus intense et est de plus accompagné d'une perte de masse plus importante dans le cas de l'argile brute TAG $(11,41 \%)$ par rapport à la fraction minérale purifiée $\mathrm{Na}^{+}$-TAG $(2,45 \%)$. Il est attribué à la décomposition de la calcite.

L'observation de la faible intensité de ce pic ainsi que la faible perte de masse $y$ étant associée dans le cas de la fraction argileuse extraite confirment que la quantité de la calcite diminue considérablement dans l'échantillon $\mathrm{Na}^{+}$-TAG. Les pics endothermiques observés à $870{ }^{\circ} \mathrm{C}$ et $890^{\circ} \mathrm{C}$ pour l'argile brute TAG et sa fraction fine 


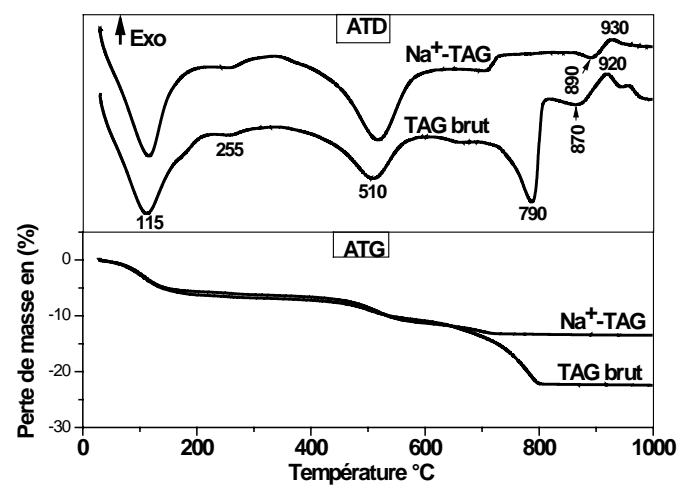

Fig. 5. Thermogrammes TG-ATD de l'argile brute (TAG) et de sa fraction fine ( $\left.\mathrm{Na}^{+}-\mathrm{TAG}\right)$.

Fig. 5. TG-DTA Thermograms of the raw clay (TG) and of its fine fraction $\left(\mathrm{Na}^{+}-\mathrm{TAG}\right)$.

$\mathrm{Na}^{+}$-TAG respectivement, associés dans les deux cas à de très faibles pertes de masses, correspondent vraisemblablement à l'achèvement de la déshydroxylation du minéral argileux. Enfin, des pics exothermiques, observés pour les deux échantillons, vers $920 \pm 10{ }^{\circ} \mathrm{C}$ correspondent à la recristallisation du minéral argileux.

L'analyse par spectroscopie IR ne révèle pas de différences très significatives entre les échantillons brut (TAG) et purifié $\left(\mathrm{Na}^{+}-\right.$ TAG). Le type de spectre FTIR obtenu, donné pour l'échantillon $\mathrm{Na}^{+}-\mathrm{TAG}$, est représenté sur la figure 6.

On distingue la présence de bandes d'absorption vers $423 \mathrm{~cm}^{-1}, 473 \mathrm{~cm}^{-1}, 530 \mathrm{~cm}^{-1}$ correspondant respectivement aux vibrations de déformation des motifs Si-O-Fe, $\mathrm{Si}-\mathrm{O}-\mathrm{Mg}$ et $\mathrm{Si}-\mathrm{O}-\mathrm{Al}$ du squelette du minéral argileux. Ce résultat indique vraisemblablement une substitution du $\mathrm{Si}^{4+}$ par $\mathrm{Al}^{3+}$ et/ou $\mathrm{Fe}^{3+}$ et/ou $\mathrm{Mg}^{2+}$ dans la couche tétraédrique. Une bande d'absorption intense, observée à $1038 \mathrm{~cm}^{-1}$, correspond aux vibrations d'élongation des liaisons Si-O. Par ailleurs, un pic relativement moins intense, observé à $910 \mathrm{~cm}^{-1}$, correspond vraisemblablement aux vibrations de déformation de la liaison $\mathrm{Al}_{2}-\mathrm{OH}$ et témoigne de la prédominance du caractère dioctaédrique du minéral smectite de l'argile TAG. Toutefois, la bande d'absorption observée à $3694 \mathrm{~cm}^{-1}$ correspondant à $\mathrm{Mg}_{3}-\mathrm{OH}$ témoigne de la présence probable de domaines trioctaédriques. Par ailleurs, en accord avec les résultats de la diffraction des rayons $X$

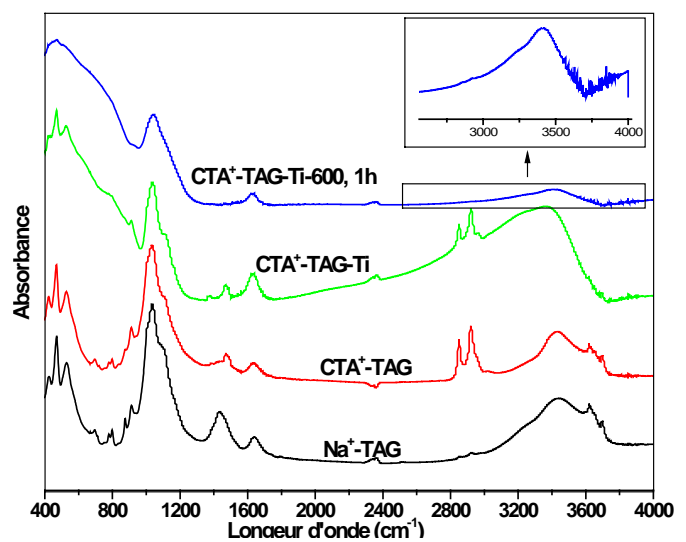

Fig. 6. Spectres FTIR des différents échantillons argileux avant et après fonctionnalisations.

Fig. 6. FTIR spectra of different clayey samples before and after functionalization.

et de l'analyse thermique, la bande d'absorption à $1444 \mathrm{~cm}^{-1}$, caractéristique de la calcite, paraissant très intense dans l'argile brute (TAG) (spectre non reporté) diminue d'intensité dans l'échantillon $\mathrm{Na}^{+}$-TAG et prouve par conséquent la diminution de sa quantité dans la fraction minérale isolée. De même, le doublet à $785 \mathrm{~cm}^{-1}$ et $790 \mathrm{~cm}^{-1}$ sont imputables au quartz. Le pic à $1630 \mathrm{~cm}^{-1}$ est attribué à l'eau physisorbée.

L'analyse par RMN du solide $\left({ }^{27} \mathrm{Al}\right.$ et ${ }^{29} \mathrm{Si}$ ) effectuée sur les échantillons TAG et $\mathrm{Na}^{+}$-TAG ne révèlent pas de différences notables. Les types de spectres obtenus sont donnés dans la figure 7 et leur dépouillement a été fait sur la base des données publiées [24]. Le spectre ${ }^{27} \mathrm{Al}$ (Fig. 7a) montre deux pics à 4,2 ppm et 68 ppm correspondant respectivement aux environnements octaédrique et tétraédrique d'Al. Toutefois, en tenant compte des intensités des deux pics, l'occupation octaédrique d'Al parait plus prépondérante. La figure $7 \mathrm{~b}$ représente le spectre RMN du ${ }^{29} \mathrm{Si}$. Deux pics intenses sont observés aux déplacements chimiques de -93 ppm et -108 ppm. Le premier pic correspond à un environnement tétraédrique $\mathrm{Si}(0 \mathrm{Al})$ du silicium, c'est-à-dire un atome de silicium entouré par trois atomes de silicium et aucun atome d'aluminium en couche tétraédrique [25]. Le déplacement à environ -108 ppm est attribué au quartz présent dans l'échantillon TAG en tant qu'impureté comme il a été également détecté par DRX et IR [26]. 

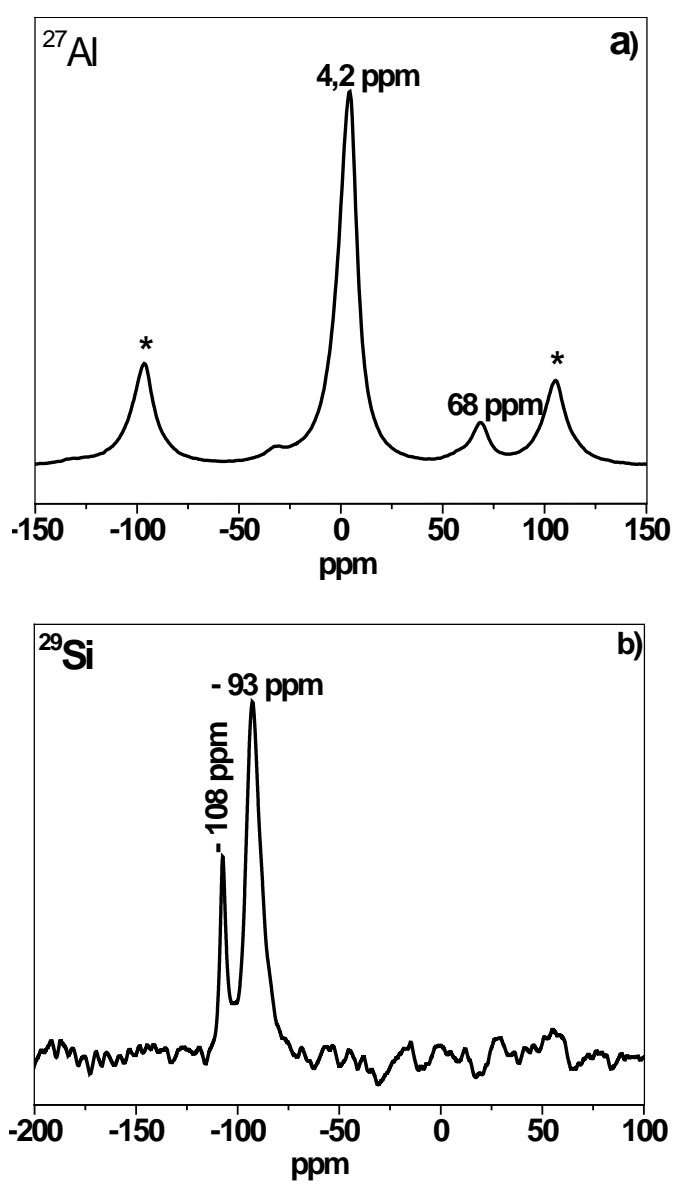

Fig. 7. Spectres RMN du solide de $\mathrm{Na}^{+}$-TAG (a) ${ }^{27} \mathrm{Al}$ et (b) ${ }^{29} \mathrm{Si}$.

Fig. 7. MAS NMR spectra of $\mathrm{Na}^{+}-\mathrm{TAG}(a)^{27} \mathrm{Al}$ et $(b)$ ${ }^{29} \mathrm{Si}$.

Les compositions chimiques des échantillons déshydratés d'argile brute (TAG) et de sa fraction fine isolée $\left(\mathrm{Na}^{+}\right.$-TAG) sont données dans le tableau 2. Ces résultats révèlent que la fraction du minéral argileux composant l'argile TAG est principalement formée de $\mathrm{Si}, \mathrm{Al}$ et Fe représentant $74,1 \%$ et $92,0 \%$ du poids total de TAG et $\mathrm{Na}^{+}$TAG respectivement. Il importe de noter que la quantité de $\mathrm{CaO}$ (correspondant à la calcite) est plus élevée dans l'argile brute TAG $(19,9 \%$ pds) en comparaison avec celle dans la fraction extraite (3,8\% pds).

En bon accord avec les résultats de la diffraction des rayons $X$ et de l'analyse thermique, ce résultat confirme parfaitement que la calcite diminue considérablement dans la fraction argileuse extraite à l'issue du traitement acide avec la solution tampon. De même, on constate que la quantité de
$\mathrm{Na}$ augmente dans l'échantillon $\mathrm{Na}^{+}$-TAG par comparaison à TAG; ce qui indique que l'homoionisation des espaces interfoliaires de la beidellite avec $\mathrm{Na}^{+}$est vraisemblablement accomplie avec succès. En supposant que le calcium est entièrement contenu dans la calcite, le nombre de cations par unité de maille de chaque élément constituant le minéral argileux est calculé sur la base de 22 atomes $\mathrm{O}$, sans tenir en compte des molécules de $\mathrm{H}_{2} \mathrm{O}$ puisque les échantillons sont préalablement déshydratés par maintien à $800{ }^{\circ} \mathrm{C}$ pendant $12 \mathrm{~h}$ [27]. La formule structurale déterminée comme $\left(\mathrm{Si}_{7,57} \mathrm{Al}_{0,43}\right)_{8}$ $\left(\mathrm{Al}_{2,75} \mathrm{Fe}_{0,73} \mathrm{Mg}_{0,38} \mathrm{Ti}_{0,14}\right)_{4} \quad\left(\mathrm{Na}_{0,37} \mathrm{~K}_{0,23} \mathrm{Mg}_{0,01}\right)$ confirme que le caractère aluminifère et dioctaédrique est prédominant dans la beidellite. De plus, en parfait accord avec les résultats de la RMN du solide, cette formule confirme la substitution de $\mathrm{Si}^{4+}$ par $\mathrm{Al}^{3+}$ dans la couche tétraédrique. De même, on note également des substitutions de $\mathrm{Al}^{3+}$ par $\mathrm{Fe}^{3+}, \mathrm{Mg}^{2+}$ et $\mathrm{Ti}^{4+}$ dans la couche octaédrique. Ces substitutions sont accompagnées d'excès de charges négatives sur les particules de la beidellite compensées par des cations interfoliaires $\left(\mathrm{Na}^{+}, \mathrm{K}^{+}\right.$et $\left.\mathrm{Mg}^{2+}\right)$.

Les observations au MET révèlent une microstructure pétaloïde caractéristique des minéraux argileux de type smectites (Fig. 8a) [28]. Le minéral argileux se présente sous la forme de feuillets dont la distance basale $d_{001}$ est d'environ $1 \mathrm{~nm}$ (Fig. 8b), en parfait accord avec les résultats de la diffraction des rayons $\mathrm{X}$ (Fig. 3).

La modification chimique des minéraux argileux par des ammoniums est connue pour conduire à l'obtention d'argiles organophiles exhibant une affinité remarquable vis-à-vis des composés organiques [15]. La figure 9 représente la variation du potentiel d'écoulement induit en fonction de la quantité de chlorure de céthyltriméthylammonium $(\mathrm{CTACl})$ ajoutée à la suspension de la beidellite $\left(\mathrm{Na}^{+}-\mathrm{TAG}\right)$. On remarque que le potentiel est initialement négatif, ce qui traduit le fait que les particules de la beidellite sont négativement chargées en raison des substitutions de $\mathrm{Si}^{4+}$ et $\mathrm{Al}^{3+}$ dans les couches tétraédrique et octaédrique du minéral argileux par des cations de valences inférieures (voir formule structurale ci-dessus). Au fur et à mesure que la quantité de $\mathrm{CTACl}$ est 
Tableau 2. Composition chimique de l'argile brute (TAG) et de sa fraction fine ( $\mathrm{Na}^{+}$-TAG) isolée. Table 2. Chemical composition of the raw clay (TAG) and its fine fraction $\left(\mathrm{Na}^{+}-\mathrm{TAG}\right)$ isolated.

\begin{tabular}{cccc}
\hline \multirow{2}{*}{ Échantillon Oxydes } & \multicolumn{2}{c}{ TAG } & $\mathrm{Na}^{+}$-TAG \\
\cline { 2 - 4 } & $\%$ masse & $\%$ masse & Cations (22 atomes O) \\
\hline $\mathrm{SiO}_{2}$ & $50,8 \pm 2,1$ & $62,0 \pm 2,4$ & 7,57 \\
$\mathrm{Al}_{2} \mathrm{O}_{3}$ & $16,9 \pm 0,3$ & $22,1 \pm 0,3$ & 3,18 \\
$\mathrm{Fe}_{2} \mathrm{O}_{3}$ & $6,4 \pm 0,2$ & $7,9 \pm 0,2$ & 0,73 \\
$\mathrm{Na}_{2} \mathrm{O}$ & $0,7 \pm 0,1$ & $1,6 \pm 0,1$ & 0,37 \\
$\mathrm{CaO}$ & $19,9 \pm 1,1$ & $3,8 \pm 0,3$ & $\approx 0$ \\
$\mathrm{MgO}$ & $2,4 \pm 0,1$ & $2,2 \pm 0,1$ & 0,39 \\
$\mathrm{~K}_{2} \mathrm{O}$ & $1,3 \pm 0,1$ & $1,5 \pm 0,1$ & 0,23 \\
$\mathrm{TiO}_{2}$ & $1,1 \pm 0,1$ & $1,5 \pm 0,1$ & 0,14 \\
$\mathrm{Total}$ & $99,5 \pm 4,1$ & $102,6 \pm 3,6$ & 12,61 \\
\hline
\end{tabular}

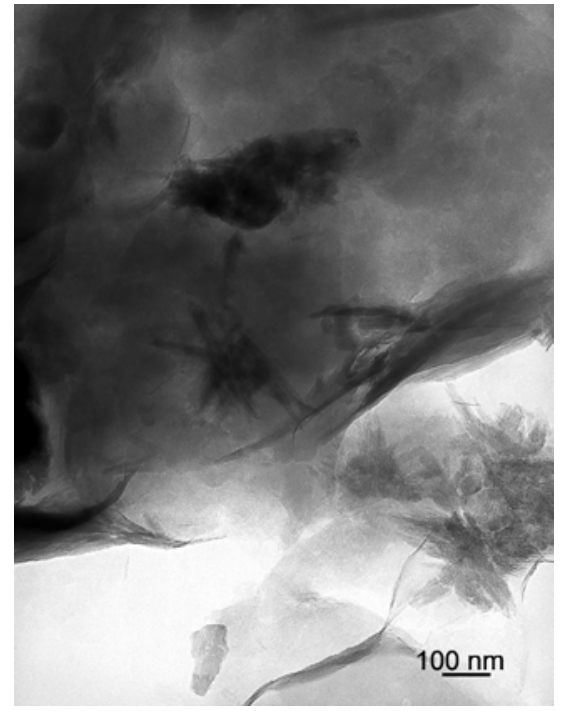

(a)

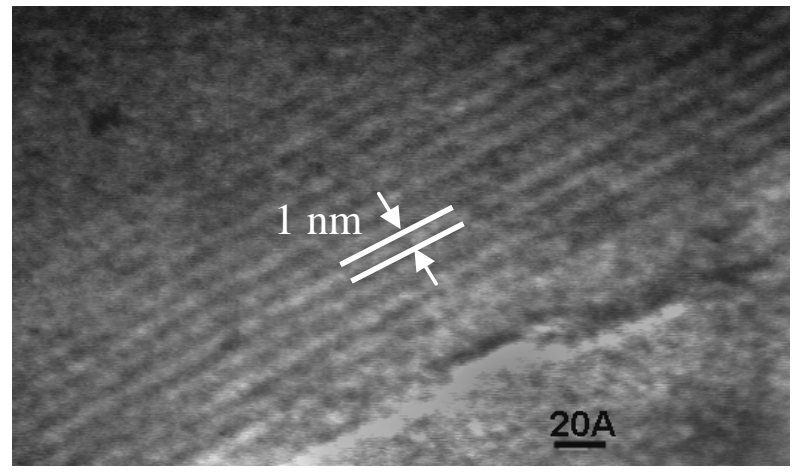

(b)

Fig. 8. Micrographies obtenues par MET montrant l'aspect pétaloïde (a) et l'empilement des feuillets (b) de la beidellite.

Fig. 8. TEM micrographs showing petalloid aspect (a) and layers stacking $(b)$ of the beidellite.

ajoutée, les charges négatives des particules de la beidellite sont progressivement compensées par les cations $\mathrm{CTA}^{+}$jusqu'au point où les particules sont de charges nulles (PCN). Le nombre de moles du surfactant ayant permis d'atteindre le PCN correspond à la capacité d'échange cationique (CEC) du minéral argileux. À cet égard, la CEC déterminée pour la beidellite est de l'ordre de 48 méq/100 g. Au-delà du PCN, l'excès de surfactant cationique inverse la charge des particules de la beidellite. Dans la présente étude, la beidellite est rendue organophile en échangeant ses cations interfoliaires par des cations de surfactant céthyltrimétylammonium (échantillon CTA ${ }^{+}$-TAG) à hauteur de 3 CEC. Le succès de l'achèvement de cette procédure est confirmé par diffraction des rayons $X$ (Fig. 10) qui montre le déplacement de la réflexion basale $d_{001}$ de l'argile initiale $(1,41 \mathrm{~nm})$ vers les petits angles dans l'échantillon modifié $\left(\mathrm{CTA}^{+}\right.$TAG) $(2,11 \mathrm{~nm}$ et 3,90 nm) indiquant l'intercalation des cations $\mathrm{CTA}^{+}$au sein des espaces interfoliaires de la beidellite. Le fait d'obtenir deux pics à l'issue de cette intercalation indique qu'elle s'est accomplie d'une manière hétérogène sous forme de bicouche pour quelques particules du minéral argileux exhibant la réflexion à 2,11 nm et sous forme paraffine pour les autres présentant la raie à 3,90 nm [24]. De même, l'analyse par spectroscopie IR révèle l'apparition de bandes d'absorption à $2854 \mathrm{~cm}^{-1}$ 


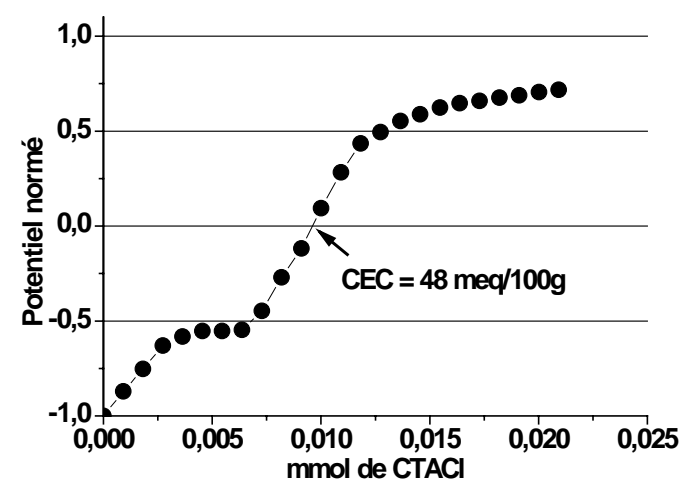

Fig. 9. Variation du potentiel normé de la suspension de $\mathrm{Na}^{+}$-TAG en fonction du nombre de moles de CTACl ajouté.

Fig. 9. Variation of the normalized potential with CTACl moles number added to $\mathrm{Na}^{+}-\mathrm{TAG}$ suspension.

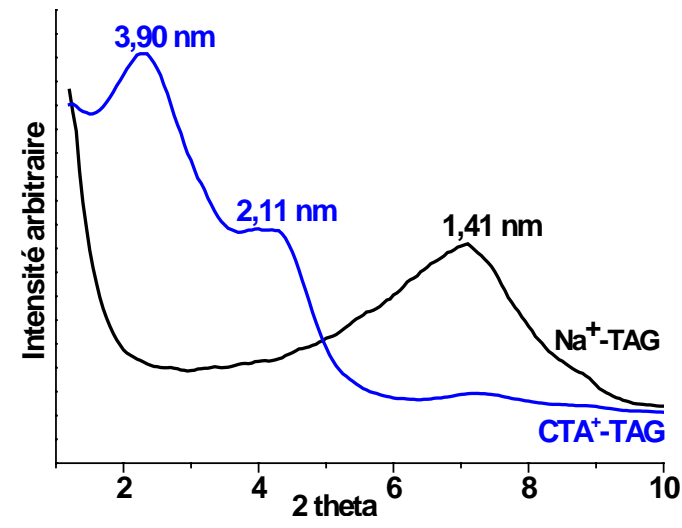

Fig. 10. Diffractogrammes de rayons $X$ des échantillons $\mathrm{Na}^{+}$-TAG et CTA+-TAG.

Fig. 10. $\mathrm{X}$ ray diagrams of $\mathrm{Na}^{+}-\mathrm{TAG}$ and $\mathrm{CT} \mathrm{A}^{+} \mathrm{TAG}$ samples.

et $2916 \mathrm{~cm}^{-1}$ caractéristiques des vibrations des groupes $-\mathrm{CH}_{2}$ - et $-\mathrm{CH}_{3}$ de la chaine alkyl du surfactant (Fig. 6).

L'échantillon de la beidellite organophile obtenu $\left(\mathrm{CTA}^{+}-\mathrm{TAG}\right)$ a fait l'objet par la suite de fonctionnalisation par $\mathrm{TiO}_{2}$ selon la procédure décrite dans la partie expérimentale.

Hormis la bande d'absorption caractéristique de $\mathrm{Si}-\mathrm{O}$ de la beidellite, celles du surfactant cationique $\mathrm{CTA}^{+}$, celle de la calcite et celle de l'eau physisorbée qui sont encore intensément observées, l'analyse IR de l'échantillon précurseur brut d'élaboration, désigné $\mathrm{CTA}^{+}-\mathrm{TAG}-\mathrm{Ti}$, révèle la disparition de quelques bandes d'absorption de la beidellite $\left(694 \mathrm{~cm}^{-1}, 877 \mathrm{~cm}^{-1}\right)$ et la forte diminution des autres bandes d'absorption $\left(423 \mathrm{~cm}^{-1}, 464 \mathrm{~cm}^{-1}, 522 \mathrm{~cm}^{-1}\right.$ et $\left.911 \mathrm{~cm}^{-1}\right) \mathrm{du}$

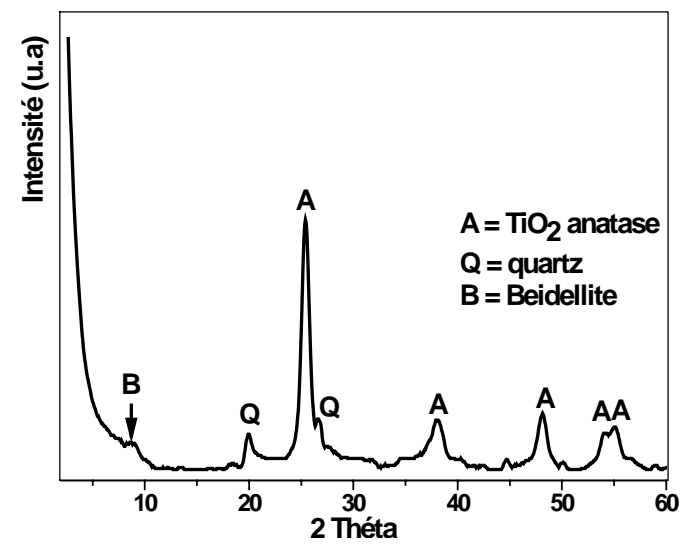

Fig. 11. Diffractogramme de l'échantillon $\mathrm{CTA}^{+}$TAG-Ti-600 enregistré après traitement thermique sous air à $600{ }^{\circ} \mathrm{C}$.

Fig. 11. $X$ ray diagram of the sample $C T A^{+}-T A G-T i-$ 600 recorded after annealing under air at $600{ }^{\circ} \mathrm{C}$.

minéral argileux (Fig. 6). Ce constat indique que les particules de la beidellite sont vraisemblablement couvertes par les espèces polymérisées hydroxo-oxo de $\mathrm{Ti}$ issues de l'hydrolyse et la polycondensation de l'isopropoxyde de Ti. L'échantillon précurseur $\mathrm{CTA}^{+}$-TAG-Ti a par la suite subi un recuit sous air à $600{ }^{\circ} \mathrm{C}$ pendant $1 \mathrm{~h}$. Ce traitement a permis l'élimination du surfactant comme le démontre clairement la disparition des bandes d'absorption - $\mathrm{CH}$ - et - $\mathrm{CH}_{2}$ - dans le spectre IR de l'échantillon CTA ${ }^{+}$-TAG-Ti600 (Fig. 6). Comme il a été précisé ci-dessus, le surfactant CTA joue seulement le rôle d'un agent promoteur de l'ancrage des nanoparticules de $\mathrm{TiO}_{2}$ aux particules du minéral argileux [14].

De plus, ce spectre IR indique également la dissimulation quasi totale des bandes d'absorption caractéristiques de la beidellite. Le diagramme de diffraction $\mathrm{RX}$ montre l'apparition des pics caractéristiques de $\mathrm{TiO}_{2}$ anatase tout comme la raie basale $d_{001}$ de la beidellite $(\approx 1 \mathrm{~nm})$ confirmant la stabilité du minéral argileux à l'issue de ce traitement à $600{ }^{\circ} \mathrm{C}$ (Fig. 11). Ce résultat important indique la stabilisation remarquable de cette phase active photocatalytiquement dans le matériau nanocomposite beidellite $\mathrm{TiO}_{2}$ développé dans cette étude. Les observations au MET et l'analyse EDX (non reportées) de l'échantillon CTA ${ }^{+}$-TAG-Ti-600 (Fig. 12) révèlent la formation de particules de $\mathrm{TiO}_{2}$ sur les feuillets de la beidellite. La 

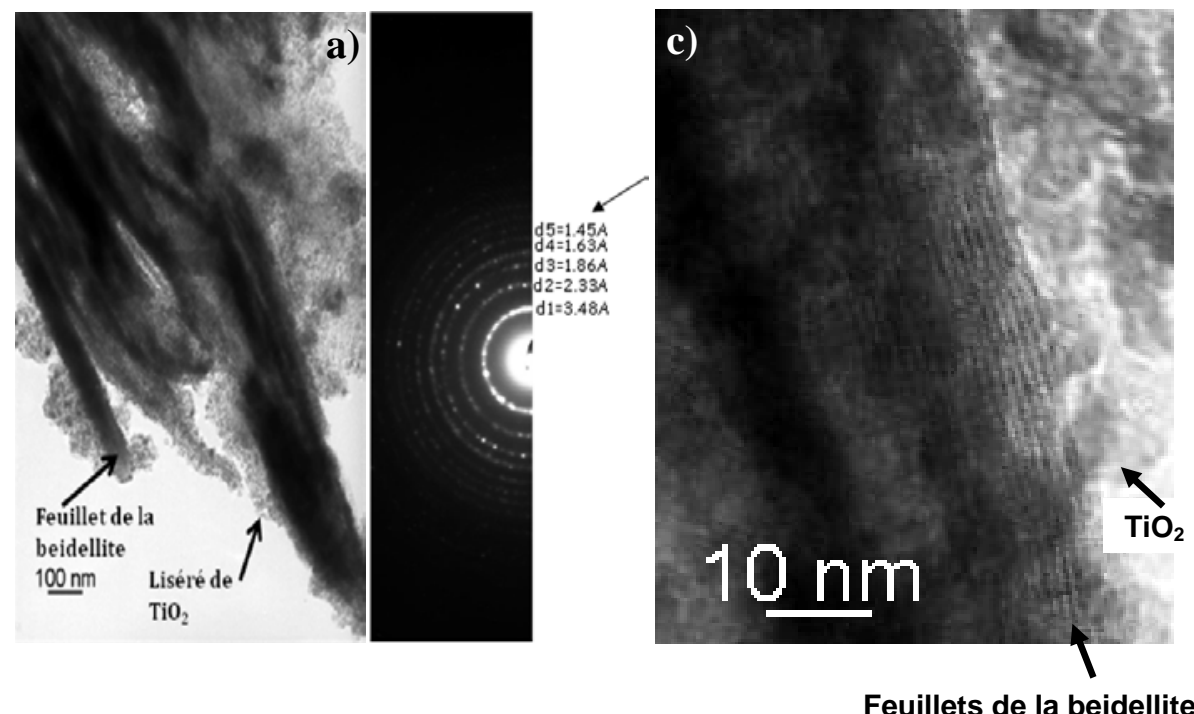

Fig. 12. Micrographies MET de 1'échantillon CTA+-TAG-Ti-600 : (a) Feuillets de la beidellite couverts par $\mathrm{TiO}_{2}$, (b) Diagramme de diffraction électronique et (c) Liséré de $\mathrm{TiO}_{2}$ supporté sur un feuillet de la beidellite.

Fig. 12. TEM micrographs of the sample CTA+-TAG-Ti-600: (a) Beidellite layers coated with $\mathrm{TiO}_{2}$, (b) electron diffraction pattern and (c) thin film of $\mathrm{TiO}_{2}$ supported on beidellite layer.

diffraction électronique effectuée sur le liseré de $\mathrm{TiO}_{2}$ couvrant les particules de beidellite révèle un diagramme d'anneaux caractéristique de l'anatase (Fig. 12b) et en bon accord avec les résultats de DRX (Fig. 11).

\section{2 Évaluation de l'activité photocatalytique}

L'activité photocatalytique des différents échantillons argileux avant et après fonctionnalisation a été testée en suspension dans une solution aqueuse du colorant OG de concentration $10^{-5} \mathrm{M}$ et de $\mathrm{pH} 7$. La figure 13 représente, pour la beidellite $\left(\mathrm{Na}^{+}-\mathrm{TAG}\right)$ et le nanocomposite $\mathrm{TiO}_{2}-$ beidellite obtenu par traitement thermique à $600{ }^{\circ} \mathrm{C}\left(\mathrm{CTA}^{+}\right.$-TAG-Ti-600), la variation de la concentration d'OG en fonction du temps d'irradiation UV.

En revanche, la courbe de variation de la concentration du colorant en fonction du temps en présence de l'échantillon précurseur avant tout traitement thermique $\left(\mathrm{CTA}^{+}-\right.$ TAG- Ti) a été obtenue en absence d'irradiation UV. On constate que l'échantillon $\mathrm{Na}^{+}-$ TAG, correspondant à la beidellite avant fonctionnalisation avec $\mathrm{TiO}_{2}$, ne manifeste aucune activité photocatalytique pas plus que d'adsorption. La forte diminution de la

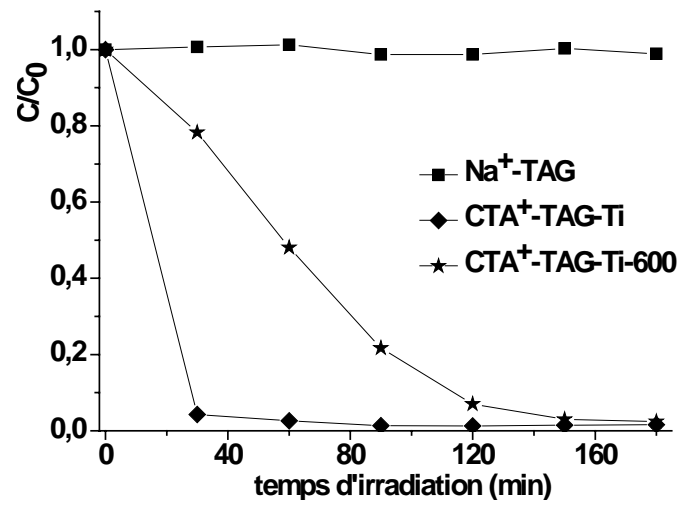

Fig. 13. Variation de la concentration de l'OG en fonction du temps en présence d'irradiation UV $\left(\mathrm{Na}^{+}-\mathrm{TAG}\right.$; CTA ${ }^{+}$-TAG-Ti-600) et sans irradiation UV (CTA ${ }^{+}$-TAG-Ti).

Fig. 13. Variation of OG concentration versus time in presence of UV irradiation ( $\mathrm{Na}^{+}-\mathrm{TAG}$; $\mathrm{CT} A^{+}-\mathrm{TAG}-$ Ti-600) and without UV irradiation (CTA+ $\left.{ }^{+}-T A G-T i\right)$.

concentration d'OG observée avec l'échantillon CTA ${ }^{+}$-TAG-Ti non recuit en absence de toute irradiation est essentiellement due à l'adsorption et non pas à la photocatalyse. En effet, comme il a été précisé ci-dessus, la modification de la beidellite avec 3 CEC du surfactant cationique $\mathrm{CTA}^{+}$inverse la charge négative de ses particules. De ce fait, et tout en étant organophile, elle adsorbe fortement les entités anioniques d'OG via 
des interactions électrostatiques [20]. En revanche, l'échantillon CTA ${ }^{+}$-TAG-Ti-600 obtenu par traitement thermique sous air à $600{ }^{\circ} \mathrm{C}$ pendant $1 \mathrm{~h}$ présente une activité photocatalytique remarquable puisqu'il permet la dégradation quasi-totale d'OG au bout de $150 \mathrm{~min}$.

\section{Conclusions}

Cette étude a permis d'isoler avec succès la fraction fine argileuse (taille des particules $<2 \mu \mathrm{m}$ ) d'une argile naturelle marocaine prélevée dans le haut Atlas, sa caractérisation, puis la préparation par sol-gel et la caractérisation d'un photocatalyseur supporté à base de $\mathrm{TiO}_{2}$ actif vis-à-vis de l'élimination de l'OG du milieu aqueux.

La fraction fine argileuse extraite est caractérisée comme étant monominérale, et elle correspond à une smectite aluminifère dioctaédrique, nommément la beidellite. Sa capacité d'échange cationique a été estimée à 48 méq/100 g.

La modification chimique de la beidellite par un surfactant cationique a été accomplie. En effet, la diffraction des RX a montré que cette modification s'opère par intercalation des cations du surfactant au sein des espaces interfoliaires de la beidellite faisant augmenter sa distance interfoliaire. De même, l'IR a révélé la présence de bandes d'absorption caractéristiques du surfactant.

La fonctionnalisation par $\mathrm{TiO}_{2}$ de la beidellite modifiée par le surfactant a été également réussie. Toutes les analyses (DRX, MET, IR) concordent pour montrer clairement des particules nanométriques de $\mathrm{TiO}_{2}$ formant des nappes couvrant les feuillets de la beidellite. Ces analyses ont révélé que la variété de $\mathrm{TiO}_{2}$ formée à l'issue du traitement thermique sous air à $600{ }^{\circ} \mathrm{C}$ pendant $1 \mathrm{~h}$ est l'anatase.

Les essais photocatalytiques effectués pour la dégradation de l'OG révèlent que le matériau nanocomposite $\mathrm{TiO}_{2}$ supporté sur la beidellite, obtenu après traitement à $600{ }^{\circ} \mathrm{C}$, est le plus actif. Par ailleurs, Cette étude se poursuivra en entreprenant une étude analytique, à l'aide de la chromatographie en phase liquide (HPLC), des différentes étapes du chemin de dégradation de l'orange G. Ainsi, il sera possible de déterminer progressivement, en fonction de la durée d'irradiation, la nature des sous-produits formés jusqu'à la minéralisation complète, autrement dit jusqu'à la formation de $\mathrm{CO}_{2}$ et $\mathrm{H}_{2} \mathrm{O}$.

Cette étude ouvre en somme des perspectives de valorisation des argiles naturelles marocaines vers leur utilisation, après fonctionnalisation par des nanoparticules de $\mathrm{TiO}_{2}$, dans des procédés photocatalytiques de dépollution des eaux. Dans de tels procédés, écologiques et propres, le comportement en terme notamment de floculation de ces nanocomposites $\mathrm{TiO}_{2}$-beidellite est plus favorable que l'utilisation directe de poudre commerciale de $\mathrm{TiO}_{2}$.

\section{Remerciements}

Cette étude a bénéficié $d u$ support du Programme d'action concertée CNRST (Maroc)/CNRS (France), projet référencé Chimie 04, du Programme de Coopération Scientifique Interuniversitaire (PCSI) de l'AUF, projet référencé 63 13PS826, du Programme d'actions intégrées Volubilis, projet référencé MA/08/185.

\section{Références}

[1] J.-M. Herrmann, Catalysis Today 53 (1999) 115-129

[2] A. Fujishima, T.-N. Rao, D.-A. Tryk, J. Photochem. Photobiol. C: Photochem. Rev. 1 (2000) 1-21

[3] Y. Paz, C. R. Chimie 9 (2006) 774-787

[4] O. Carp, C.L. Huisman, A. Reller, Prog. Solid State Chem. 32 (2004) 33-177

[5] P.K.-J. Robertson, J. Cleaner Prod. 4 (1996) 203-212

[6] M. Houari, M. Saidi, D. Tabet, P. Pichat, H. Khalaf, Am. J. Appl. Sci. 2 (2005) 1136-1140

[7] J. Liu, M. Dong, S. Zuo, Y. Yu, Appl. Clay Sci. 43 (2009) 156-159

[8] E.P. Reddy, L. Davydov, P. Smirniotis, Appl. Catalysis B 42 (2003) 1-11

[9] X.-Y. Chuan, M. Hirano, M. Inagaki, Appl. Catalysis B 51 (2004) 255-260

[10] J.M. Hermann, J. Matos, J. Disdier, C. Guillard, J. Laine, S. Malato, J. Blanco, Catalaysis Today 54 (1999) 255-265

[11] J. Feng, X. Hu, P.L. Yue, H.Y. Zhu, G.Q. Lu, Chem. Eng. Sci. 58 (2003) 679-685

[12] T. An, J. Chen, G. Li, X. Ding, G. Sheng, J. Fu, B. Mai, K.-E. O'Shea, Catalysis Today 139 (2008) 69-76 
[13] M. Nieto-Suárez, G. Palmisano, M.L. Ferrer, M.C. Gutiérrez, S. Yurdakal, V. Augugliaro, M. Pagliaro, F. del Monte, J. Mater. Chem. 19 (2009) 2070-2075

[14] P. Aranda, R. Kun, M.A. Martin-Luengo, S. Letaïef, I. Dékány, E. Ruiz-Hitzky, Chem. Mater. 20 (2008) 84-91

[15] Z. Bouberka, A. Khenifi, F. Sekrane, N. Bettahar, Z. Derriche, Chem. Eng. J. 136 (2008) 295-305

[16] L. Khalfallah Boudali, A. Ghorbel, D. Tichit, B. Chiche, R. Dutartre, F. Figueras, Microporous Materials 2 (1994) 525-535

[17] B. Rhouta, H. Kaddami, J. Elbarqy, M. Amjoud, L. Daoudi, F. Maury, F. Senocq, A. Maazouz, J.-F. Gerard, Clay minerals 43 (2008) 393-404

[18] A. Jada, H. Debih, M. Khodja, J. Petroleum Sci. Eng. 52 (2006) 305-316

[19] C. Sarantopoulos, A.N. Gleizes, F. Maury, E. Puzenat, C. Guillard, J.-M. Herrmann, Applied Catal. B : Environmental 91 (2009) 225233
[20] L. Bouna, B. Rhouta, M. Amjoud, A. Jada, F. Maury, L. Daoudi, F. Senocq, Applied Clay Science 48 (2010) 527-530

[21] K. Hofstadler, B. Rupert, S. Novalic, G. Heisler, Environ. Sci. Technol. 28 (1994) 670674

[22] T. Holtzapffel, Société Géologique du Nord 12 (1985) 15-43

[23] G. Parthasarathy, B.M. Choudary, B. Sreedhar, A.C. Kunwar, R. Srinivasan, American Mineralogist 88 (2003) 1983-1988

[24] G. Lagaly, Advances in Colloid and Interface Science 114 (2005) 189-204

[25] S. Komarneni, C.A. Fyfe, G.J. Kennedy, H. Strobl, J. Am. Ceram. Soc. 69 (1986) 45-47

[26] S. Komarneni, C.A. Fyfe, G.J. Kennedy, Clays and clay Minerals 34 (1986) 99-102

[27] M. Rautureau, S. Caillère, S. Hénin, les argiles, 2nd edn, Éditions Septima, Paris, 2004

[28] H. Chamley, Clay Sedimentology, Springer, Berlin, 1989 